\title{
ADEM post-Sars-CoV-2 infection in a pediatric patient with Fisher-Evans syndrome
}

\author{
Maria Laura Manzo ${ }^{1}$. Cristina Galati ${ }^{1}$. Cristina Gallo ${ }^{2}$. Giuseppe Santangelo ${ }^{3}$ - Antonio Marino ${ }^{1} \cdot$ Fulvio Guccione $^{1}$. \\ Renata Pitino ${ }^{3} \cdot$ Vincenzo Raieli $^{3}$
}

Received: 27 March 2021 / Accepted: 5 May 2021 / Published online: 12 May 2021

(C) Fondazione Società Italiana di Neurologia 2021

\begin{abstract}
Introduction Sars-CoV-2 is a single-strained RNA virus belonging to Coronaviridae's family. In pediatric age, the majority of patients is asymptomatic; however, several neurological manifestations associated with Sars-CoV-2 infection have been detected in a percentage of cases ranging from 17.3 to $36.4 \%$. Acute disseminated encephalomyelitis (ADEM) has been recently included among the potential complications of Sars-Cov2 infection. The available data regarding pediatric patient show only one case.

Case report We present a case regarding a 6-year-old patient suffering from Fisher-Evans syndrome who was given sirolimus and thalidomide therapy. After 10 days since the first positive nasopharyngeal swab for Sars-CoV-2, in which he had no symptoms, he presented an episode of generalized tonic-clonic seizure with spontaneous resolution. The patient underwent MRI which showed the typical picture of acute disseminated encephalomyelitis. His clinical course was favorable, with a good response to cortisone therapy and a progressive improvement of the neuroradiological and electroencephalographic picture.

Conclusions According to our knowledge, this is the second case of an acute disseminated encephalomyelitis following SARS$\mathrm{CoV}-2$ infection in a pediatric patient, characterized by monosymptomatic onset, in which the immunosuppressive therapy practiced for the Fisher-Evans syndrome has probably contributed to a favorable evolution of ADEM, in contrast to other case described in the literature.
\end{abstract}

Keywords Headache $\cdot$ Epilepsy $\cdot$ ADEM $\cdot$ Fisher-Evans Syndrome $\cdot$ Autoimmune disease

\section{Introduction}

Sars-CoV-2 is a single-strained RNA virus belonging to Coronaviridae's family. The clinical picture associated with this type of air-borne infection is extremely variable and heterogeneous. However, accordingly to the literature, the main manifestation seems to be interstitial pneumonia [1]. Despite in pediatric age, the majority of patients is asymptomatic; various neurological manifestations associated with SarsCoV-2 infection are described in percentages ranging from 17.3 to $36.4 \%$. Among these we can list: headache, snoring, ageusia, anosmia, vomiting, ataxia, epileptic seizures,

Renata Pitino

renapiti@gmail.com

Vincenzo Raieli

vincenzoraieli@gmail.com

cgalati.cg@gmail.com

Cristina Gallo

crilla.gallo@gmail.com

Giuseppe Santangelo

giuseppe.santangelo@arnascivico.it

Antonio Marino

antoniomarino94@tiscali.it

Fulvio Guccione

fulvio.guc@gmail.com
Child Neuropsychiatry School, University of Palermo, Piazzale delle Cliniche, 90100 Palermo, Italy

2 U.O.C Neuroradiology ARNAS Civico, Piazzale Nicola Leotta 4, 90100 Palermo, Italy

3 Child Neuropsychiatry Unit, ISMEP-P.O. Cristina-ARNAS Civico, Via Dei Benedettini 1, 90100 Palermo, Italy 
encephalopathy, stroke, myopathy, Guillan Barrè Syndrome, and acute hemorrhagic necrotizing encephalopathy [2]. ADEM has been recently included among the possible complications of Sars-Cov2 infection; one concerns a pediatric patient [3-5]. In this short observations, we report a second case of ADEM regarding a 6-year-old patient with Sars-Cov2 infection, showing an insidious clinical presentation.

\section{Case report}

We report a 6-year-old male patient suffering from FisherEvans syndrome, characterized by autoimmune hemolytic anemia, immune thrombocytopenia (ITP), and/or autoimmune neutropenia by May 2019, and was given a sirolimus and thalidomide therapy with clinical benefit [6]. The child came to our observation for the occurrence of a first brief epileptic seizure by generalized tonic-clonic semiology with spontaneous resolution. Ten days before the occurrence of the epileptic seizure, the patient tested positive for SARS-CoV-2 through nasopharyngeal swab; meanwhile no symptoms of COVID-19 had occurred. There were no focal neurological deficits. During the hospitalization, he presented only mild headache, and he did not exhibit any further critical episodes. Anti-MOG and anti-AQ4 antibodies were negative. CSF analysis showed absence of cells and proteins. Culture and panel for encefalitis/meningitis resulted negative. Otherwise, CSF underlined the presence of $\operatorname{IgG}, \operatorname{IgM}$, and IgG type 3 oligoclonal bands and expression of intrathecal synthesis. A brain MRI study, acquired 3 days after the neurological symptoms (epileptic seizure) onset, showed bilateral and asymmetrical cortical and subcortical supra- and subtentorial cerebral parenchymal lesions with corpus callosum involvement, characterized by hyperintensity in long TR sequences and no perifocal edema, ranging from a few millimeters to about $2,5 \mathrm{~cm}$ of diameter, the largest one, in the left parietal lobe. Almost all lesions and a subarachnoid sulcus, adjacent to the largest lesion, show contrast-enhancement in $\mathrm{T} 1 \mathrm{w}$ sequence; there is also thickening and post-contrast enhancement of the ipsilateral free margin of the tentorium (Fig. 1).

Fig. 1 First brain MRI study (GE 1,5T). (A, B, C) T2-FLAIR sequence shows hyperintense lesions in the right cerebellar hemisphere, cortical-subcortical cuneus gyrus of the right parietal lobe, left side of the corpus callosum and corona radiata, cortical-subcortical inferior left parietal gyrus. (D) Post-contrast T1w shows signal increase in the inferior left parietal gyrus lesion

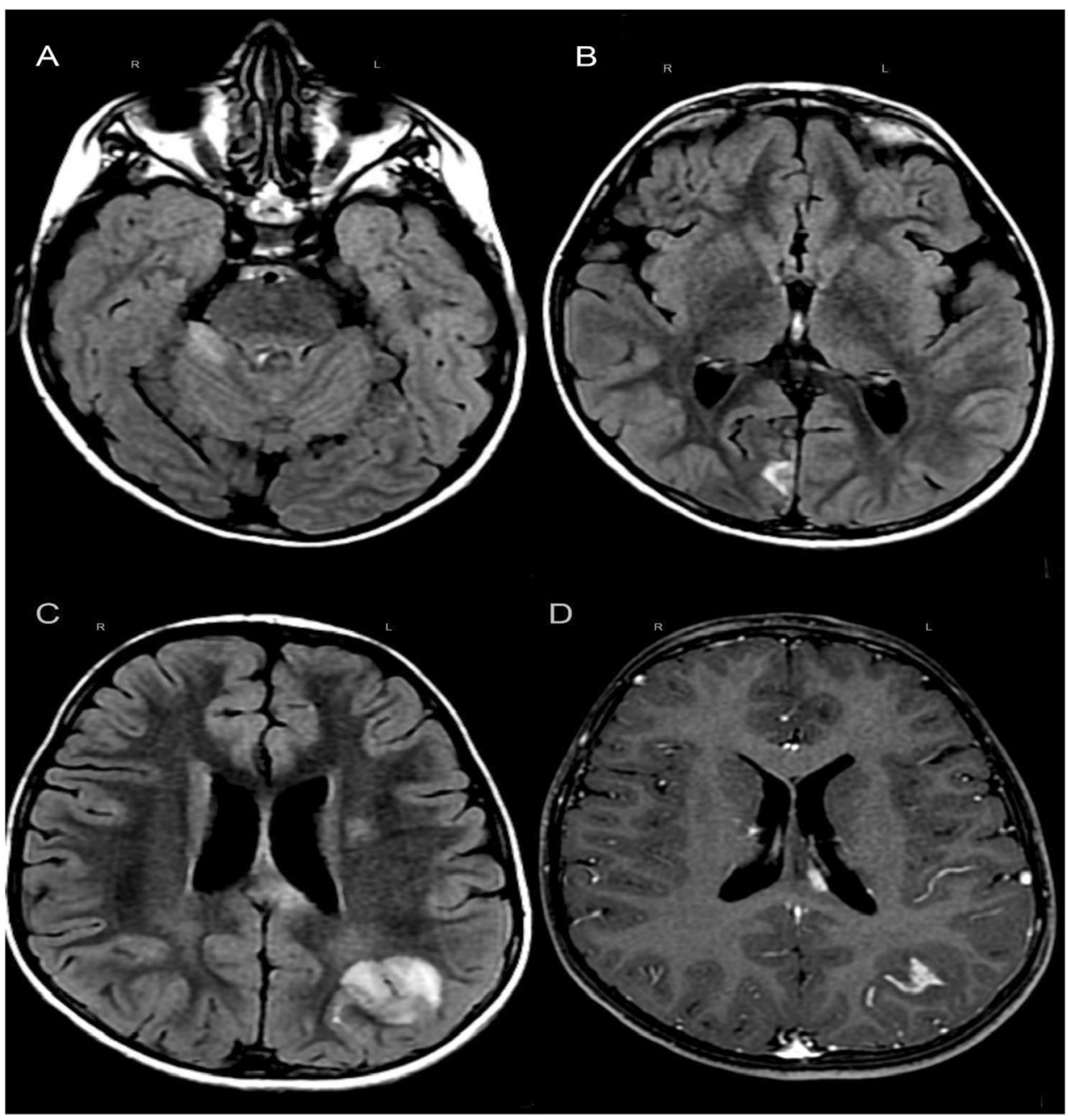


Electroencephalogram (EEG) showed slow posterior bilateral activity more expressed on the right. During hospitalization, he was treated with methylprednisolone ev ( $30 \mathrm{mg} / \mathrm{kg} / \mathrm{die})$ for 5 days, followed by progressive steroid dose tapering. About a month later, a check-up confirmed the good clinical status. A follow-up brain MRI examination, performed 25 days later, reported dimensional and signal hyperintensity reduction of the multiple lesions previously described with slight residual contrast enhancement of the largest lesion; of adjacent sulcus and of the other lesions was not evident anymore.

\section{Discussion}

ADEM is a rare immune-mediated demyelinating disease of the central nervous system, triggered by viral or bacterial infections or environmental stimuli, in a genetically susceptible host. ADEM is usually monophasic, occasionally biphasic or multiphasic. The pathogenesis is not completely understood: it is considered an autoimmune disorder whose trigger is represented by myelin antigens, such as myelin basic protein, proteolipid protein, or MOG, which share antigenic determinants with infecting pathogens. Lesions associated with ADEM are usually multiple, bilateral, and asymmetric and show featuring of demyelination. They are often large and with poorly defined margins and usually localized in the deep and subcortical white matter. Our patient analogously had bilateral and asymmetrical supra- and subtentorial cerebral parenchymal lesions extending from few millimeters to about $2.5 \mathrm{~cm}[7,8]$. Nonetheless, he was also affected by Fisher-Evans syndrome, which is characterized by autoimmune hemolytic anemia, immune thrombocytopenia (ITP), and/or autoimmune neutropenia, and he was treated with sirolimus and thalidomide [6]. In the available literature, there are no cases of ADEM associated with sirolimus or thalidomide therapy. However, only one case of posterior reversible encephalopathy syndrome (PRES) has been described in a patient with sirolimus-induced brain lesions [9]. In our patient, the pathogenetic hypothesis of PRES was excluded after performing brain MRI. He was diagnosed with Sars-CoV-2 infection before ADEM. Considering the latency time, compatible with such response, the lack of antibody titers related to other possible recent viral infections, and the presence of an autoimmune reaction evidenced by the oligoclonal bands in the CSF, we hypothesized that the ADEM could have been triggered by the Sars-CoV-2 infection in a child already suffering from an autoimmune condition. Our case of ADEM following Sars-CoV-2 infection appears to be very different from both the adults and the pediatric cases reported in literature [3-5], which showed the typical manifestations of suspected Sars-CoV-2 infection at the onset, worsened by a recent Coxackie virus infection, and much more severe neurological symptoms requiring observation in the intensive care unit. Conversely, our case was completely asymptomatic for Covid-19. The only clinical finding was a generalized epileptic seizure, which could be framed as idiopathic due to the age, the full recovery, the lack of CT findings, and the typical post-critical course. The diagnostic process was further investigated through brain MRI and subsequent CSF examination after the anamnestic data of recent SARS-CoV2. Despite the moderate brain involvement, the symptoms remained poor and with a very encouraging evolution, unlike the other pediatric case. We hypothesize that the monosymptomatic favorable clinical course was promoted by the immunomodulatory action of sirolimus and thalidomide drugs that the patient already practiced for FisherEvans syndrome. Moreover, these drugs have also been recently proposed for the treatment of Sars-CoV-2 infection, thanks to their anti "cytokinic storm" action.[10]

\section{Conclusion}

According to our knowledge, this is the second case of pediatric post-Sars-CoV-2 acute disseminated encephalomyelitis described in the literature. Our case is characterized by a monosymptomatic onset, in which the immunosuppressive therapy practiced for the Fisher-Evans syndrome has probably contributed to the favorable evolution of ADEM. Furthermore, in our clinical case, there is a dissociation between the few neurological manifestations and the neuroradiological alterations present in brain MRI examination. This suggests the importance of an accurate diagnostic work-up in the presence of Sars-CoV-2 positivity despite minimal neurological symptoms.

Acknowledgement Many thanks to Andrea Santangelo for English review of manuscript.

\section{Declarations}

Conflict of interest The authors declare no competing interests.

Ethical approval Not applicable

\section{References}

1. Christy A (2020) COVID-19: a review for the pediatric neurologist. J Child Neurol 35(13) SAGE Publications Inc.:934-939. https:// doi.org/10.1177/0883073820939387

2. Correia AO, Feitosa PWG, Moreira JL d S, Nogueira SÁR, Fonseca RB, Nobre MEP (2020) Neurological manifestations of 
COVID-19 and other coronaviruses: a systematic review. Neurol Psychiatry Brain Res 37. Elsevier GmbH:27-32. https://doi.org/10. 1016/j.npbr.2020.05.008

3. Novi G, Rossi T, Pedemonte E, Saitta L, Rolla C, Roccatagliata L, Inglese M, Farinini D (2020) Acute disseminated encephalomyelitis after SARS-CoV-2 infection. Neurol Neuroimmunol Neuroinflammation 7(5):2-5. https://doi.org/10.1212/NXI. 0000000000000797

4. De Miranda Henriques-Souza A., De Melo A., De Aguiar Coelho Silva Madeiro B, Freitas L, Rocha-Filho P, Goncalves F Acute disseminated encephalomyelitis in a COVID-19 pediatric patient. https://doi.org/10.1007/s00234-020-02571-0/Published

5. McCuddy M, Kelkar P, Zhao Y, Wicklund D (2020) Acute demyelinating encephalomyelitis (ADEM) in COVID-19 infection: a case series. medRxiv 5:5-8. https://doi.org/10.1101/2020.07.15. 20126730

6. S. Audia, N. Grienay, M. Mounier, M. Michel, and B. Bonnotte, "Evans' syndrome: from diagnosis to treatment," J Clin Med., 9, 12, 3851, 2020, doi: 10.3390/jcm9123851
7. Leake J, Albani S, Kao A et al (2004) Acute disseminated encephalomyelitis in childhood: epidemiologic, clinical and laboratory features. Pediatr Infect Dis J 23(8):756-764. https://doi.org/10. 1097/01.inf.0000133048.75452.dd

8. Cole J, Evans E, Mwangi M, Mar S (2019) Acute disseminated encephalomyelitis in children: an updated review based on current diagnostic criteria. Pediatr Neurol 100:26-34. https://doi.org/10. 1016/j.pediatrneurol.2019.06.017

9. Gheith O, Cerna M, Halim MA et al (2017) Sirolimus-induced combined posterior reversible encephalopathy syndrome and lymphocytic pneumonitis in a renal transplant recipient: case report and review of the literature. Exp Clin Transplant 15:170-174. https:// doi.org/10.6002/ect.mesot2016.P36

10. Atrah HI (2020) Alternative management of Covid-19 infection. Scott Med J 65(3):72-75. https://doi.org/10.1177/ 0036933020941497

Publisher's note Springer Nature remains neutral with regard to jurisdictional claims in published maps and institutional affiliations. 